\title{
Pursuit-evasion predator-prey waves in two spatial dimensions
}

\author{
V. N. Biktashev \\ Department of Mathematical Sciences, University of Liverpool, Liverpool L69 7ZL, UK \\ J. Brindley \\ Department of Applied Mathematics, University of Leeds, Leeds LS2 9JT, UK
}

A. V. Holden

School of Biomedical Sciences, University of Leeds, Leeds LS2 9JT, UK

M. A. Tsyganov

Institute of Theoretical and Experimental Biophysics, Pushchino, Moscow Region 142290, Russia

(Dated: September 22, 2018)

\begin{abstract}
We consider a spatially distributed population dynamics model with excitable predator-prey dynamics, where species propagate in space due to their taxis with respect to each other's gradient in addition to, or instead of, their diffusive spread. Earlier, we have described new phenomena in this model in one spatial dimension, not found in analogous systems without taxis: reflecting and self-splitting waves. Here we identify new phenomena in two spatial dimensions: unusual patterns of meander of spirals, partial reflection of waves, swelling wavetips, attachment of free wave ends to wave backs, and as a result, a novel mechanism of self-supporting complicated spatio-temporal activity, unknown in reaction-diffusion population models.
\end{abstract}

\section{INTRODUCTION}

To describe spatio-temporal dynamics of populations one needs, apart from local interaction of populations, to invoke some mechanism of spatial interaction, e.g spread of individuals in space. Often this is done in terms of diffusion, which is a macroscopic way of describing random, non-directed movement of individuals. This makes a population dynamics model a reaction-diffusion system, and pattern formation in reaction-diffusion systems is well researched. However, living organisms do not necessarily move randomly, and often show some directed movement in response to exogeneous factors, a behaviour usually described as taxis. Macroscopic representation of taxis yields equations different from reaction-diffusion systems, and pattern formation in such models has been studied much less. Our aim is to elucidate some new pattern formation mechanisms specific to this type of model.

In our previous work 1, 2], we have described new phenomenon observed numerically in a reaction-diffusion-taxis model, with excitable predator-prey local kinetics 3]. In addition to or instead of diffusion, we introduced taxis of each species on the other's gradient: predators pursuing prey and prey escaping the predators. We chose excitable rather than more traditional limit-cycle predator-prey dynamics, as it is methodologically easier to deal with solitary population waves than with wavetrains, and identified some new features that are typical in our excitable pursuitevasion model, but unknown or very rare in reaction-diffusion models of similar systems. These include ability of propagating waves to penetrate through each other or reflect from impermeable boundaries (rather than simply annihilate, as is typical for reaction-diffusion waves), split and emit backward waves.

This was done in models with one spatial dimension. In most ecological applications, however, two spatial dimensions are be more realistic. In reaction-diffusion systems it is well known that two-dimensional models can demonstrate a much wider variety of nontrivial solutions than one-dimensional. Thus, in the present paper, we describe phenomenology observed in two-dimensional simulations of our pursuit-evasion excitable predator-prey model. As far as we are aware, this is the first study of this sort, so rather than giving an exhaustive description, we have identified phenomena that are qualitatively different from what is observed in two-dimensional reaction-diffusion excitable systems.

\section{THE MODEL}

We consider a two-dimensional version of the model, studied earlier in [1, 2]:

$$
\begin{aligned}
& \frac{\partial P}{\partial t}=f(P, Z)+D \nabla^{2} P+h_{-} \nabla(P \nabla Z), \\
& \frac{\partial Z}{\partial t}=g(P, Z)+D \nabla^{2} Z-h_{+} \nabla(Z \nabla P),
\end{aligned}
$$


where $P(x, y, t)$ and $Z(x, y, t)$ are biomass densities of the prey and predator populations, $h_{-}$is the coefficient determining the taxis of prey down the gradient of predators (evasion), $h_{+}$is the coefficient determining the taxis of predators up the gradient of prey (pursuit), $D$ is their diffusion coefficient, assumed equal for simplicity, and $f$ and $g$ are "kinetic" terms, describing local interaction of the species with each other. We assume that this local interaction takes the Truscott-Brindley [3] form,

$$
\begin{aligned}
& f(P, Z)=\beta P(1-P)-Z P^{2} /\left(P^{2}+\nu^{2}\right), \\
& g(P, Z)=\gamma Z P^{2} /\left(P^{2}+\nu^{2}\right)-w Z,
\end{aligned}
$$

which describes excitable kinetics. Equations (12) are solved numerically with the following parameter values: $\nu=$ 0.07, $\beta=1, w=0.004$. Parameters $\gamma$ and $D$ have been used in two fixed combinations, $(\mathrm{A}) \gamma=0.01, D=0.04$, and (B) $\gamma=0.016, D=0$. Parameters $h_{-}, h_{+}$varied between simulations. From one-dimensional simulations, described in [2], we know that in (A), purely reaction diffusion waves without taxis terms $\left(h_{-}=h_{+}=0\right)$ are possible, where as in (B), such waves are not possible.

All two dimensional simulations were performed in a rectangular domain $(x, y) \in\left[0, L_{x}\right] \times\left[0, L_{y}\right]$, with no-flux boundary conditions,

$$
\left.\frac{\partial(P, Z)}{\partial x}\right|_{x=0, L_{x}}=\left.\frac{\partial(P, Z)}{\partial y}\right|_{y=0, L_{y}}=0 .
$$

In figures representing the results of the simulations, we designate the domain size as $L_{x} \times L_{y}$, choice of $\gamma, D$ combination as (A) or (B), the values of taxis coefficients as $\left(h_{-}, h_{+}\right)$, and where the panels are presented with a regular time interval, it is given as $\Delta t$.

\section{PURSUIT-EVASION WAVES IN ONE DIMENSION}

\section{A. Mechanism of propagation}

It is useful to first recapitulate the one-dimensional results, and we start with propagation of solitary waves. Figure 1 shows typical profiles of steadily propagating waves in a reaction-diffusion system and in a reaction-taxis system, and schematically illustrates various mechanism involved. In the reaction-diffusion wave (panel a), the density of predators changes only slightly through the front. The propagation of the wave front is mostly due to interaction between diffusion and nonlinear local dynamics of the prey: influx of prey due to the diffusion triggers the prey-escape mechanism, when the prey multiply faster than the predators and therefore grow unchecked until reaching the carrying capacity of the habitat. Between the front and the back of the wave the prey and predators are in quasi-equilibrium, the predators slowly consuming the prey and growing themselves. On the back, the concentration of the predators is so large that the high concentration of prey is no longer sustainable, and their dynamics are such that the transition to their low state is sudden, and is therefore also influenced by their diffusion, as at the front. The sequence of events in the reaction-taxis wave is entirely different (panel b). The advancing front of prey attracts the predators in the retrograde direction. This leaves space relatively free of predators, which triggers the prey-escape mechanism. Another mechanism, driving the prey forward, is evasion, i.e. their taxis away from the predators. This feedback loop of prey evading predators and predators pursuing prey leads to spatial oscillations of both (similar to the way the feedback loop of prey benefiting predators and predators harming prey tends to produce their temporal oscillations). The similarity with reaction-diffusion waves is that the front peaks when the prey reach their high quasi-equilibrium. The difference, apart from oscillatory character of the taxis front, is that the density of predators does not remain nearly constant during the front, but instead shows a marked tip followed by a rise, due to predators first moving back towards advancing prey, and then stopping that movement when the gradient of prey is over. The events on the waveback are also quite different. There we observe an advancing wave of predators pursuing prey, and the receding wave of prey escaping predators. This recession of prey is enhanced also by the fact that they are consumed by pursuing predators. Thus the position of the back is not directly related to the time taken by predators to grow to a certain concentration or to consume a certain numbers of prey, but rather, by time and space needed for the pursuit-evasion dynamics to form the back structure. As a result, the duration of the taxis wave is much shorter than that of the diffusion wave.

\section{B. Mechanism of reflection}

Figure 2 illustrates the process of reflection of the taxis wave from an impermeable boundary. When the front of the wave arrives at the boundary, the system there switches to the high-prey state, close to the carrying capacity 
of the habitat, i.e. the maximal stationary density of prey if predators are absent, which in our model is $P=1$ $\left(t=t_{0}+200\right)$. This is similar to what happens in a reaction-diffusion wave, and in both cases is mainly due to the nonlinear dynamics of the prey population. The difference comes when the back of the excitation wave approaches the boundary $\left(t=t_{0}+200 \ldots t_{0}+400\right)$, because in the taxis wave, unlike the diffusion wave, the back of the wave profile is formed by prey evading the peak of predators following them, rather than by consumption of prey by predators. As a result, in the taxis wave, the density of predators at the back of the wave is insufficient to reduce prey density to a low level during the time that the tail actually lasts. Thus, when the evading prey are stopped by the impermeable boundary, the predators catching up with them do not consume them all. Instead, a substantial density of prey survives $\left(t=t_{0}+400\right)$. Those prey that survive after the maximum of the predator density has passed them escape in the retrograde direction $\left(t=t_{0}+600\right)$, and if their number is large enough, initiate a new backward propagating wave $\left(t=t_{0}+800\right)$.

Thus we see that the mechanism of reflection of taxis waves is entirely different from known mechanisms of reflection of reaction-diffusion waves, e.g. positive overlap of oscillatory tails of the colliding waves discussed in [4], which required a very close proximity of the local dynamics to the Hopf bifurcation. In that case the reflection property was restricted to a narrow region in the parameter space. With the present mechanism the reflection is rather robust, both in terms of local dynamics, and of taxis and diffusion coefficients [2].

\section{SPIRAL WAVES}

Amongst the most ubiquitous, dramatic and intensively studied phenomena observed in 2D reaction-diffusion excitable systems are re-entrant excitation waves, commonly known as spiral waves [5]. They are observed in a variety of experimental systems, and in an even greater variety of mathematical models, that includes spatially extended mathematical models of predator-prey interaction of the reaction-diffusion type [6]. Their significance stems from the fact that they can occur in an event of a wave break in a propagating excitation for whatever reason, and thus serve as a specific route spatio-temporal chaos. Thus, we have started to study details of reaction-diffusion-taxis waves in $2 \mathrm{D}$ through the effect that the taxis terms have on the spiral waves.

\section{A. Very small taxis can destabilize spirals}

Figure 3 shows a typical rigidly rotating spiral wave solution, observed in the purely reaction-diffusion model. From the behaviour of 1D solutions [2, 2], qualitatively new behaviour in 2D based on 1D phenomenology (e.g. reflection from the boundaries) is expected when the taxis terms are large enough in comparison with coefficient $D(=0.04$ in our simulations). The relevant values are of the order of 1 , which corresponds to comparable fluxes generated by the diffusion and the taxis terms, at the values of the variables at around the stable equilibrium. Comparison of fluxes gives a reasonable way to compare the relative significance of taxis and diffusion terms, as $h_{ \pm}$are coefficients of nonlinear terms and have different dimensionality from that of $D$.

Figure 4 shows the behaviour of the reaction-diffusion-taxis spiral wave at values of the taxis coefficients much smaller than those at which reflection happens. Already these small taxis terms change the behaviour of the spiral very significantly, as, with the same local kinetics as the stationary spiral of fig. 3 it now meanders wildly. This kind of non-stationary behaviour of spiral waves is not unknown in excitable media, where the transition from steady to meandering rotation is usually associated with change of the parameters of the reaction; here we see such a transition for the same reaction parameters but with the spatial terms altered.

As the taxis coefficients increase, their effect on the spiral wave dynamics increases further. Figure [5] shows that the spiral wave meander becomes so extended that the spiral soon drifts out of the medium and annihilates at the boundary. This happens at taxis coefficients that are still much smaller than those needed for quasi-soliton behaviour.

\section{B. Peculiar behaviour of the tip: waltzing spiral}

As the 1D studies have shown, if taxis terms are present, waves can propagate without any diffusion. Figure 6 shows a strikingly different behaviour of a spiral wave in such a system in 2D. Apparently, the tip of the spiral periodically switches between two meta-stable states, in one of which it curls up as in a typical spiral wave in a strongly excitable reaction-diffusion system, and in the other it propagates along a straight line orthogonally to the front of the wave, as happens in an excitable system on the verge of excitability. This results in a "slow waltz" trajectory, when the spiral makes $5 / 4$ of a turn, then moves along a straight line, then makes another $5 / 4$ of a turn, and so on. In total, the spiral makes exactly five turns per each round of, and in the same direction as the meander trajectory. 
The ratio $1: 5$ is not unique, as spiral waves in reaction diffusion systems can demonstrate a continuous spectrum of these ratios as kinetic parameters change 7, 8]. It fits well with theoretical considerations based on symmetry considerations [9, 10, 11, 12, 13], except that an exact four-fold symmetry should, from the viewpoint of that theory, be non-typical. Apparently in our case its appearance should be attributed to a resonance with the slight anisotropy of the numerical discretization. The mechanism of the meander appears more exotic, in which the tip trajectory consists of alternating pieces of very small and large curvature, where the high-curvature pieces form more than one full turn of the spiral wave. As far as we are aware this kind of spiral wave meander has not been seen in reaction-diffusion systems.

\section{ALTERNATIVE BEHAVIOUR OF THE WAVE TIPS}

In reaction-diffusion excitable systems, if an excitation wave is broken, its tip will either grow ("germinate"), protrude and curl up into a spiral wave, if the medium is "strongly excitable", or it may shrink and retract, if it is "weakly excitable". Figure 7 shows an example of a reaction-taxis excitable system, where neither takes place. The tip of a broken wave protrudes, but does not curl up into a spiral; instead new characteristic phenomena are observed.

\section{A. Wavetip swelling}

See fig. $7 t=40 \ldots 60$. The consequence of the wavebreak protruding may be seen as "swelling" of the tip, as it goes through a "blobbing" stage, when an initially small oval-shape region of medium is excited, which subsequently grows and reforms into circular expanding wave stage. A phenomenological interpretation of this swelling phenomenon follows from the empirical observation that it usually happens when the evasion coefficient is large compared to the pursuit coefficient. Thus the prey near the wavebreak have the capacity to escape from the predators sideways. A subpopulation of prey then finds itself in a region relatively free from predators. In this predator-free zone prey start multiplying intensively and form a circular expanding region. As there is no chasing wave of predators inside this circular region, the high-prey state ends only when the predators multiply to large densities, taking much longer than in a standard reaction-taxis propagating wave where the end of the prey wave is determined by the chasing wave of predators. So the swollen tip can grow to form a large patch of prey, before turning into an annular shape wave, when the back-structure of the taxis wave is formed. Subsequently, the predator wave catches up with the prey and the circular expanding region develops into a annulus-shaped expanding wave.

\section{B. Self-attachment}

See fig. 7 $t=60 \ldots 240$. After the tip swelling phase, no wavebreak can be identified based on the distribution of the prey population. Nevertheless, a wavebreak, or tip, can be formally defined, e.g. as an intersection of suitable isolines of the prey and predator populations, as illustrated on fig. 8 . For topological reasons, such a tip cannot disappear, but has to move continuously until reaching the medium boundary or annihilating with another tip of the opposite chirality. Such a "tip" is found near where the circular wave, newly born from the swollen tip, touches the back of the mother wave. Now the whole old+new wave can be seen as a single wave, a free end of which is attached somewhere to its own back. This could not happen in a reaction-diffusion medium, because of the refractory period behind an excitation wave. In terms of the present population dynamics system, the refractory period is characterised by such high density of predators that triggering a prey-escape mechanism is not feasible. The difference with taxis excitation waves is that they are not characterised by significant difference in the predators' density before and after the wave. To put it briefly, the prey wave finishes not just when all prey are consumed by predators, but rather when those that are not consumed have escaped forward. Similarly, the predators' wave finishes not when all predators die out because of starvation, but rather when all predators have moved forward in the pursuit of prey. Thus the equilibrium concentration of predators and prey behind the wave establishes rather quickly and due to a mechanism quite different from reaction-diffusion waves. As a result, there is little or no "refractory tail" behind a taxis wave, and this makes the self-attachment of a wave's tip to its own back possible.

The structure of the attachment site is illustrated in more detail on fig. 8 The high prey regions of the mother wave and the circular wave are connected, whereas their high predator regions are disjoint, or, more precisely, connected by an isthmus of much smaller concentration of predators than elsewhere, because the predators at the junction have been attracted backwards by the circular wave. Note that the attached wave tip has to move considerably faster than a plane solitary wave, as it participates simultaneously in the movement of the mother wave, and the circular wave, which moves at an angle to the mother wave. One more reason for such faster movement is the unusual nonlinear 
dispersion relationship for taxis waves, where speed increases as period decreses. This property is illustrated on fig. 9] which shows velocity of waves propagating on a one-dimensional interval with periodic boundary conditions. Initially a wave was started on an interval of a large length $L$. This length then was decreased in small steps, after each of which sufficient time was allowed for the circulating wave to approach its stationary velocity $v(L)$, which was then recorded, and the next decrease of $L$ was made. To circumvent the interpretation difficulty related to the difference of spatial scales in diffusion and taxis terms, we plot not the absolute velocity, but the velocity relative to the velocity of a solitary wave, $v(\infty)$. For the same reason, the horizontal coordinate on fig. 9 is not the spatial period of waves $L$, but their temporal period $T$ calculated as $L / v(L)$.

Another way to view the mechanism of the attachment of the free end is through kinetics of the prey, which multiply more efficiently because of the gap in the predators' population at the site of the junction; the wave grows in the gap rather than propagates into it (in fact, of course, prey growth is an essential component of the wave propagation, so both explanations are valid).

\section{PARTIAL REFLECTION}

As we have already mentioned, at appropriate taxis coefficients, one-dimensional waves can reflect from the boundaries and penetrate through each other. In two dimensions, there are new aspects characterising impact of the waves with boundaries and each other, not available in one dimension: the curvature of the waves at the moment of impact, and angle of incidence. In reaction-diffusion systems, the the wave propagation velocity is known to depend on the curvature of the wave. We have found that in our reaction-taxis model, the curvature, or angle of incidence, or both, affect the result of the impact, i.e. whether the wave will be reflected from the medium boundary or another wave, or will annihilate at it. A representative example is shown on fig. 10. A circular wave was initiated at an asymmetrically located site within a rectangular region, so its distances to all four boundaries were different. The result is that the wave has mostly annihilated at the northern and western boundaries (except small parts of the wave at the further ends of those boundaries), which the wave reached first while having a higher curvature. Then the wave has mostly reflected from the southern and eastern boundaries, which it reached later while having a lower curvature. The waves reflected from the eastern and southern boundaries partly annihilate on impact, namely, where their collision is more head-on. Meanwhile, where their collision is more slantwise, the waves penetrate each other. One more factor that can affect the outcome of the collision, is the "age" of the propagating wave. This is related to delicate asymptotic properties of the taxis waves which will be considered in more detail elsewhere [14]. For the present paper, the important observation is that a colliding wave may either annihilate or penetrate/reflect in the same medium, depending on the local details of the collision. Therefore, the outcome of collision may be different for different parts of the same colliding wave, which means that the wave will be broken.

\section{SELF-SUPPORTING ACTIVITY}

We have identified two specifically two-dimensional phenomena that can happen to pursuit-evasion waves: partial reflection/penetration, and tip swelling. These two phenomena can work together to generate a self-supporting spatiotemporal activity. The typical sequence of events is: partially reflected/penetrated waves are broken, i.e. have free ends. Unlike waves in reaction-diffusion systems, these free ends do not curl up into spirals, but swell and produce circular waves with the free end eventually attached to the back of its mother wave. These waves interact with each other and with medium boundaries, where some of them are partially penetrated/reflected, i.e. broken, which leads to new swollen tips, and so this sequence of events repeats. We have, as a result, a spatio-temporal "chaos". The mechanism described is different from that known before in reaction-diffusion excitable systems, or indeed in any other nonlinear spatially distributed systems. Examples of such self-supporting activities at different values of parameters are shown on the series of figures, 11] 15 We present a broad collection of simulations, to show that this mechanism is not restricted to a narrow region of parameters but is rather typical. In figs. 11] 14] and [15]initial conditions were in the form of a single spot that produced initially a circular wave, while in figs. 12 and 13 initial conditions were in the form of an artificially broken plane wave, which in a reaction-diffusion system would generate a spiral wave. Figure 14 is for kinetics A, which supports waves, particularly spiral waves in a reaction-diffusion system without taxis, whereas the rest of this set are for kinetics B, with various combinations of taxis coefficients, which does not support either spiral or any other type of waves without taxis. Kinetics A of fig. 14] shows strong waves, with relatively thick stripes of prey and very large swollen tips, whereas kinetics B in fig. [15] produces relatively thin waves with rather modest swollen tip (their traces can be noticed e.g. at the $t=620$ panel). The self-supporting activity can last long, but not necessarily for ever, as there is always a possibility that all waves will annihilate on collisions so that activity survives, as happens in fig. 15] Obviously the probability of that is higher in a smaller system. For instance, in fig. [15] the 
square symmetry of the problem means that the effective size of the system is eight time smaller than the area of the square.

\section{CONCLUSIONS}

We have demonstrated that two-dimensional excitable reaction-diffusion-taxis systems can exhibit new properties, unavailable in one-dimensional systems of the same kind, and different from two-dimensional purely reaction-diffusion systems with the same kinetics. Even relatively small taxis terms can make spiral waves very unstable in conditions where they would be stable without such terms. If the taxis (pursuit/evasion) terms are large enough, compared to diffusion, to cause quasi-soliton behaviour in $1 \mathrm{D}$, then the $2 \mathrm{D}$ behaviour is entirely different and is no longer dominated by processes of spiral waves generation and births, as is typical for reaction-diffusion systems. Instead, a new type of self-supporting activity takes place, which is mediated by three qualitatively new types of events,

- Partial reflection of waves from boundaries, or their partial penetration through each other, which produces broken waves, and

- "Swollen tips", i.e. circular wave sources, produced by free ends of broken waves.

- Attachment of free ends of broken waves to the wavebacks.

The first of these phenomena is due to sensitive dependence of quasi-soliton behaviour on the circumstances of collision. The second is due to the increased possibility of the sideward escape of prey from the head of the propagating taxis wave near the wave break, when their mobility is higher than that of the predators. The third is due to short supply of predators at the site of attachment, which allows prey to multiply quicker to seal the gap. All three phenomena are related to the peculiar feature of the reaction-taxis waves, the virtual absence of the refractory tail, which is a prominent feature of reaction-diffusion excitation waves.

The value of this study is twofold. First, this illustrates the importance of taking into account of directed movement of species in modelling spatially distributed interacting populations, as this can produce completely different phenomena from those that occur in reaction-diffusion systems. Second, this is interesting in a broader nonlinear science context, as an example of a new type of nonlinear dissipative waves, with some properties similar to those known before, and some completely new.

As we noted earlier, quasi-soliton behaviour can be observed in some reaction-diffusion excitable systems if its kinetics are close to a Hopf bifurcation [4]. In two spatial dimensions, such systems also demonstrate unusual types of behaviour, such as converging, concave spirals [15, 16, 17], but again in very limited regions of parameter space.

\section{Acknowledgments}

This study was supported in part by EPSRC grant GR/S08664/01 (UK) and RFBR grant 03-01-00673 (Russia).

[1] M.A. Tsyganov, J. Brindley, A.V. Holden, and V.N. Biktashev. Quasi-soliton interaction of pursuit-evasion waves in a predator-prey system. Phys. Rev. Lett., 91:218102, 2003.

[2] M. A. Tsyganov, J. Brindley, A. V. Holden, and V. N. Biktashev. Properties of population taxis waves in a predator-prey system with pursuit and evasion, 2004. submitted to Physica D.

[3] J. E. Truscott and J. Brindley. Equilibria, stability and excitability in general class of plankton population models. Phil. Trans. R. Soc. A, 347:703-718, 1994.

[4] O. A. Mornev, O. V. Aslanidi, R. R. Aliev, and L. M Chaylakhyan. Soliton regimes in the fitzhugh-nagumo model: Reflection of colliding pulses of excitation. Doklady Biophys., 346-348:21-23, 1996.

[5] A. T. Winfree. Spiral waves of chemical activity. Science, 1972.

[6] J. A. Sherratt, X. Lambin, C. J. Thomas, and T. N. Sherratt. Generation of periodic waves by landscape features in cyclic predator-prey systems. Proc. Roy. Soc. Lond. ser. B, 269:327-334, 2002.

[7] A. T. Winfree. Varieties of spiral wave behaviour - an experimentalist's approach to the theory of excitable media. Chaos, 1:303-334, 1991.

[8] D. Barkley and I. G. Kevrekidis. A dynamical systems approach to spiral wave dynamics. Chaos, 4(3):453-460, 1994.

[9] D. Barkley. Euclidean symmetry and the dynamics of rotating spiral waves. Phys. Rev. Lett., 72:164-167, 1994.

[10] V. N. Biktashev, A. V. Holden, and E. V. Nikolaev. Spiral wave meander and symmetry of the plane. Int. J. of Bifurcation and Chaos, 6(12):2433-2440, 1996. 
[11] C. Wulff. Bifurcation theory of meandering spiral waves. In Z. Parisi, S. C. Müller, and W. Zimmermann, editors, Nonlinear Physics of Complex Systems - Current Status and Future Trends. Springer Verlag, Berlin, 1996.

[12] M. Nicol, I. Melbourne, and P. Ashwin. Euclidean extensions of dynamical systems. Nonlinearity, 14:275-300, 2001.

[13] M. Golubitsky, V. G. LeBlanc, and I. Melbourne. Meandering of the spiral tip: An alternative approach. Journal of Nonlinear Science, 7(6):557-586, 1997.

[14] M. A. Tsyganov and V. N. Biktashev. Half-soliton interaction of population taxis waves in predator-prey systems with pursuit and evasion, 2004. submitted to Phys. Rev. E.

[15] O. A. Mornev, O. V. Aslanidi, and L. M. Chailakhyan. Solitonic mode in the FitzHugh-Nagumo equations: Dynamics of a rotating spiral wave. Doklady Academii Nauk, 353:682-686, 1997.

[16] O. A. Mornev, O. V. Aslanidi, and I. M. Tsyganov. Soliton-like regimes, echo, and concave spiral waves in mathematical models of biological excitable media. Macromolecular Symposia, 160:115-122, 2000.

[17] O. A. Mornev, I. M. Tsyganov, O. V. Aslanidi, and M. A. Tsyganov. Beyond boundaries of the Kuramoto-Zeldovich theory: stably rotating concave spiral waves and their link to the echo phenomenon. JETP Lett., 77:319-325, 2003. 


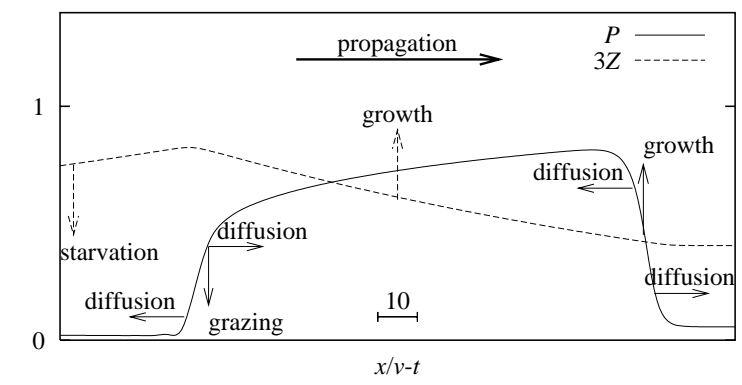

(a)

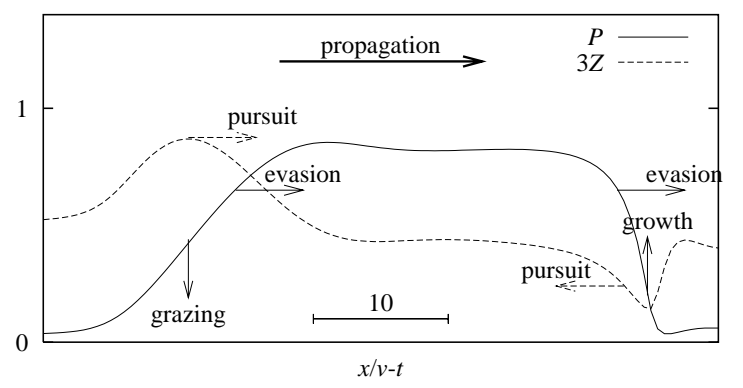

(b)

FIG. 1: A stationary propagating wave in (a) reaction diffusion system $\left(D=0.04, h_{ \pm}=0\right)$, (b) reaction-taxis system $(D=0$, $\left.h_{-}=2, h_{+}=1\right)$, and $\gamma=0.01$ and other parameters standard in both cases. Arrows illustrate the main factors affecting the dynamics of the front and the back of the wave. These include: prey dynamics (growth and decrease due to grazing pressure) in both (a) and (b), diffusion of prey in (a), and taxis (pursuit by predators and evasion by prey) in (b).

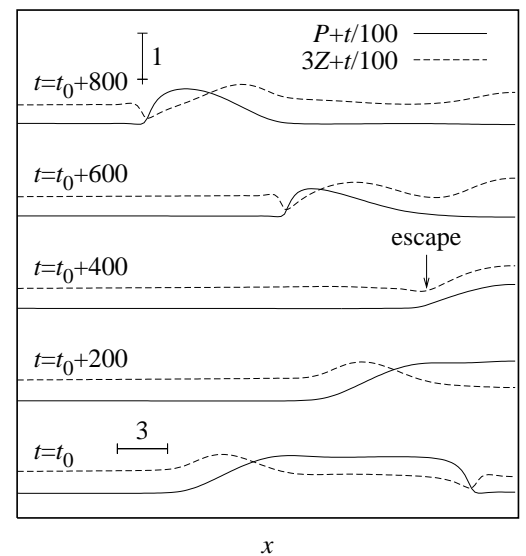

FIG. 2: Reflection of a taxis wave from an impermeable boundary $\left(D=0, h_{-}=2, h_{+}=1, \gamma=0.01\right)$.
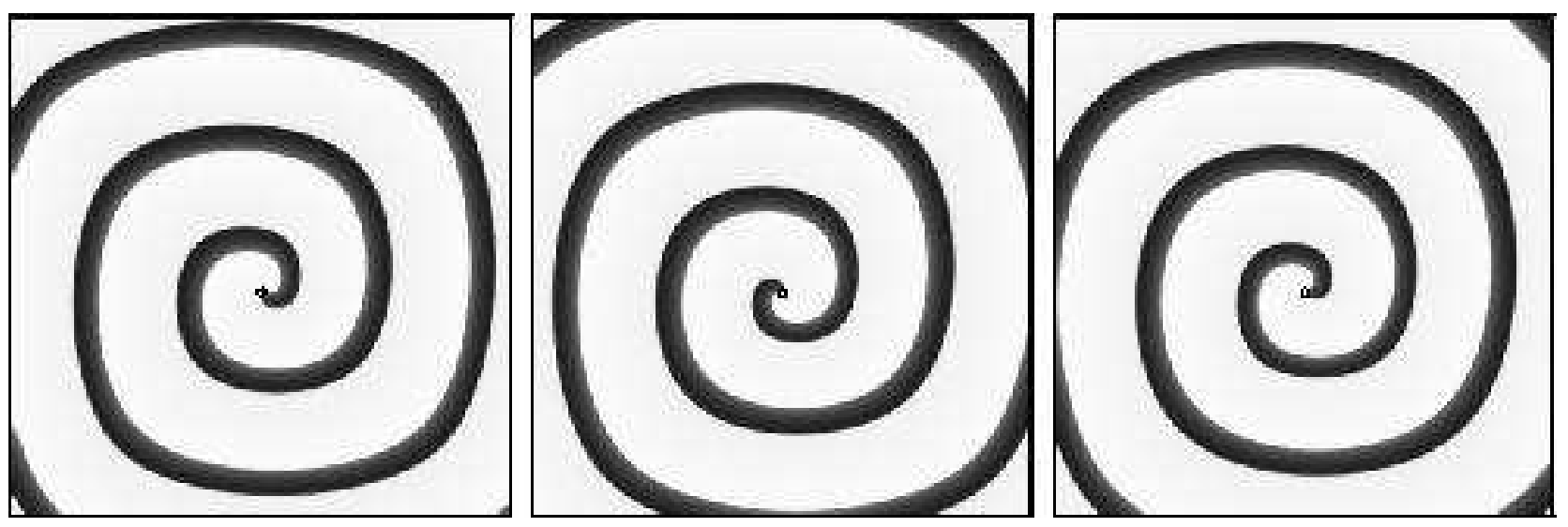

FIG. 3: Spiral wave in a purely diffusive medium, (A) $200 \times 200(0,0), \Delta t=150$. Here and on the next figures with spiral waves, the trajectory of the tip is superimposed. The tip is defined as a point where $P=0.49$ and $Z=0.21$; normally, there is only one such point per tip of a spiral wave. 

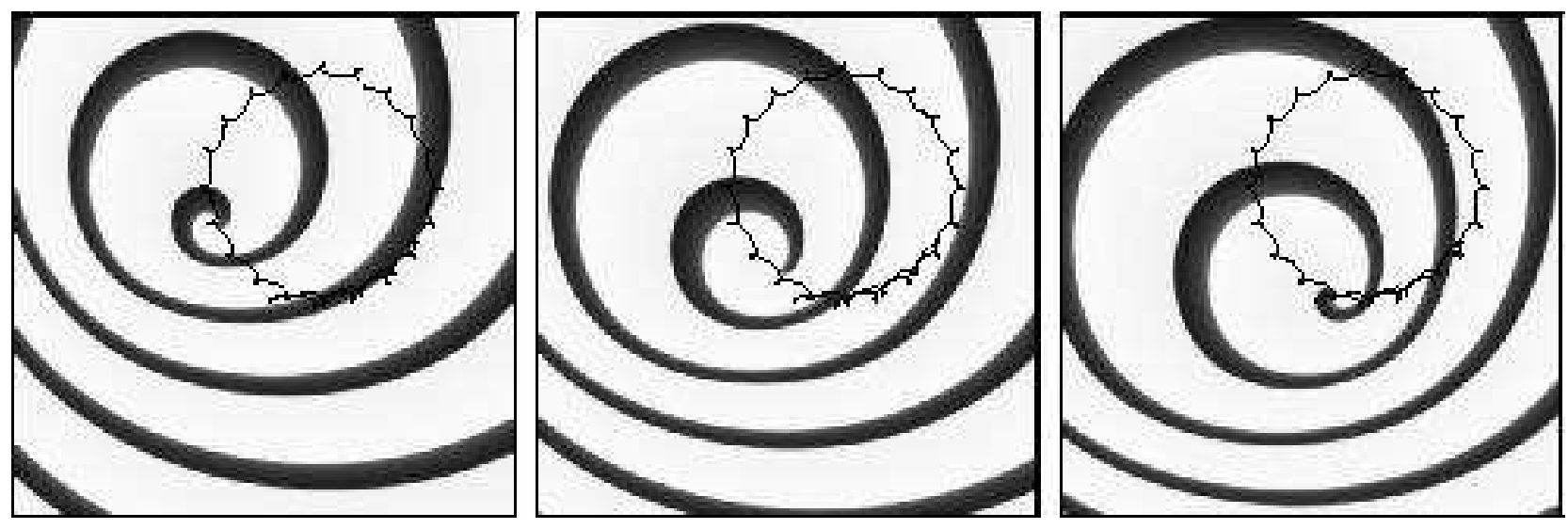

FIG. 4: Meandering spiral in a medium with small taxis coefficients, (A) $200 \times 200(0.05,0.05), \Delta t=400$.
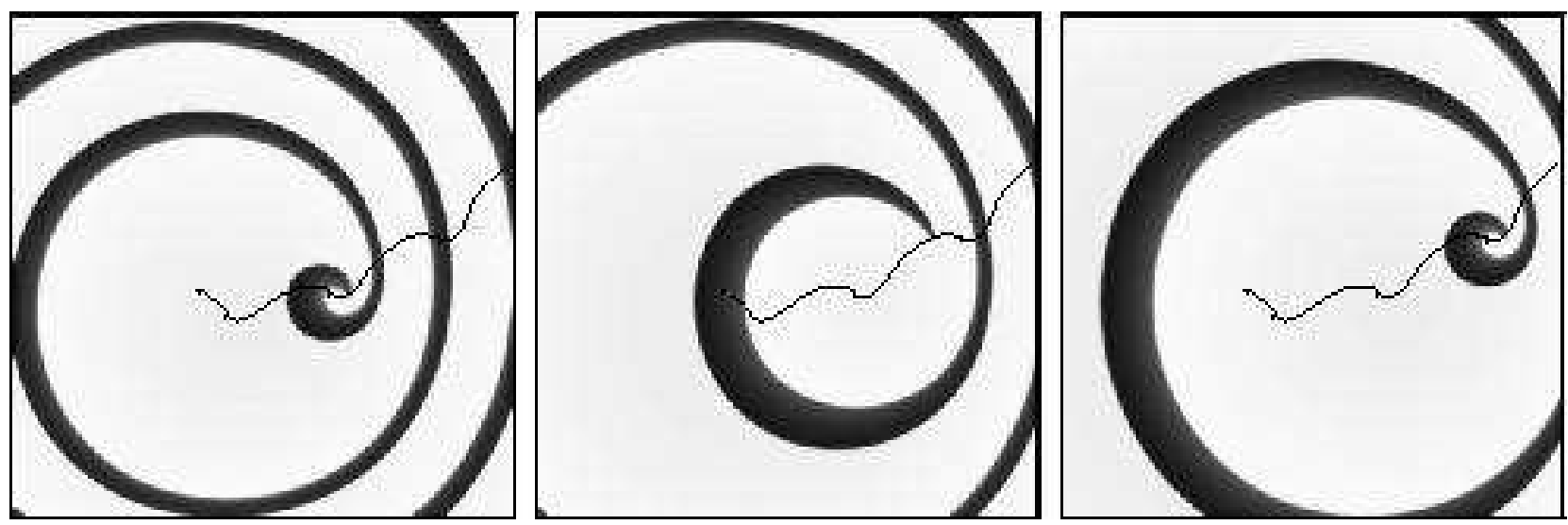

FIG. 5: Unstable spiral at larger taxis coefficients, (A) $200 \times 200(0.1,0.1), \Delta t=225$.
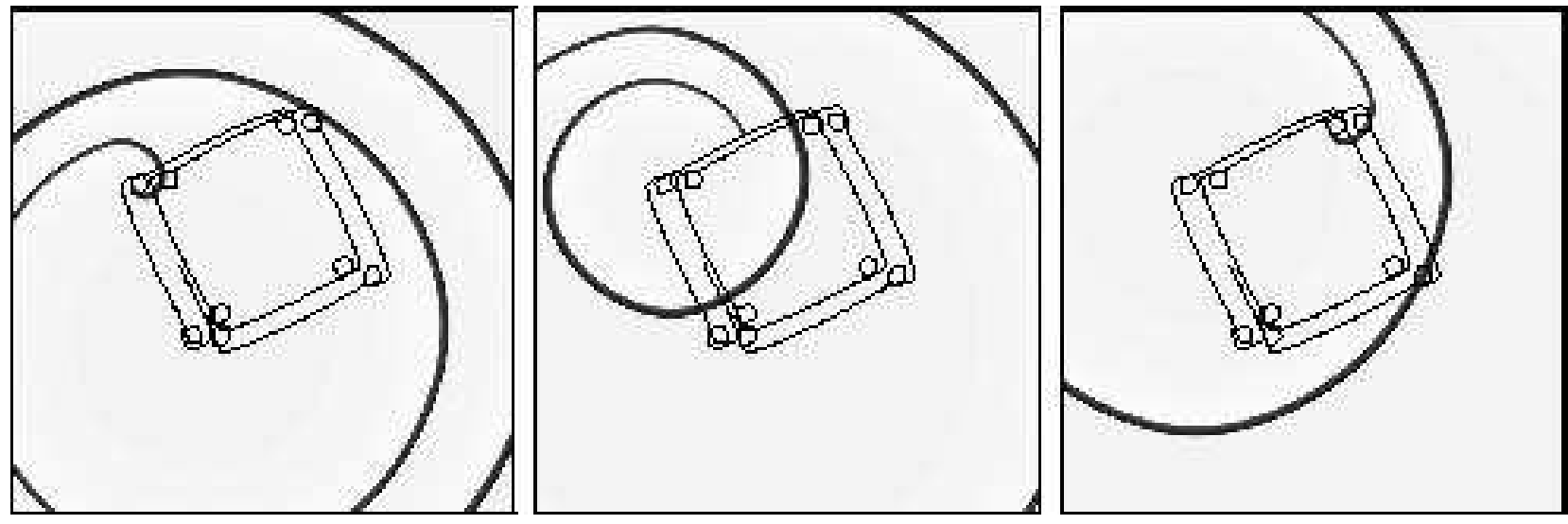

FIG. 6: Waltzing spiral wave, (B) $250 \times 250(5,0), \Delta t=450$. 


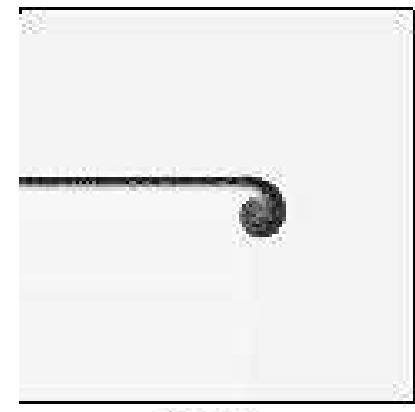

$t=4$ 사

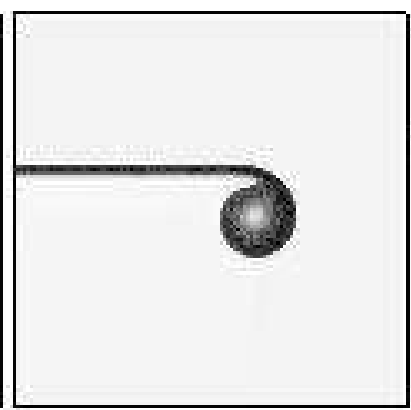

$\mathbf{t}=\mathbf{6} \mathbf{d}$

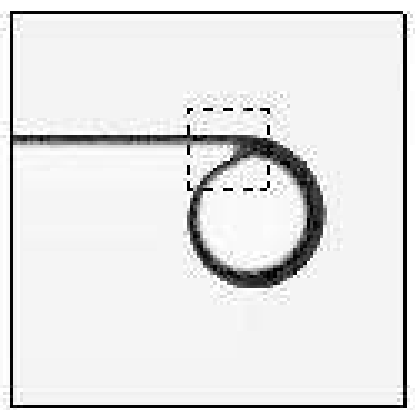

$t=1(k)$

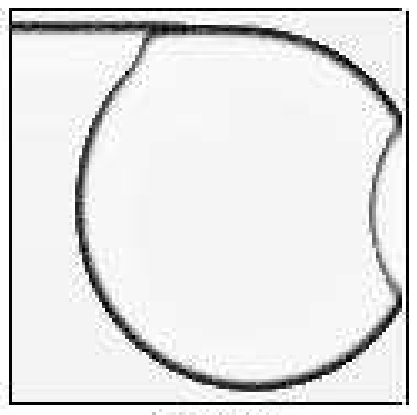

$t=24 k$

FIG. 7: Wavebreak that does not lead to spiral wave initiation, (B) $250 \times 250(5,1)$. At $t=60$ : predators have multiplied in the center of the circular prey patch, ending the "swollen tip" phase. At $t=100$ : dashed rectangle is shown in detail below on fig. 8 At $t=240$ : a piece of the wave has reflected from the eastern boundary.
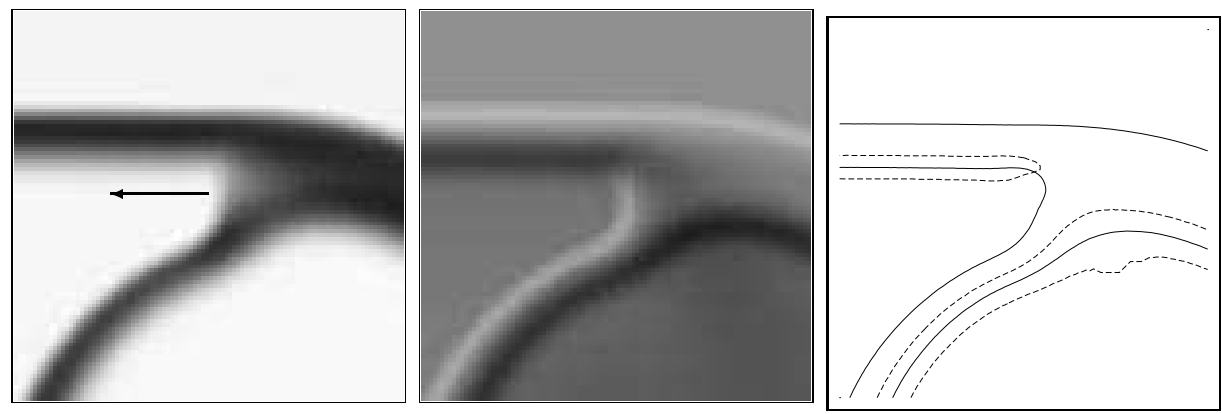

FIG. 8: Structure of the self-touching (B) $250 \times 250(5,1)$. (a) distribution of $P$, (b) distribution of $Z$, (c) isolines $P=$ const (solid) and $Z=$ const (dashed).

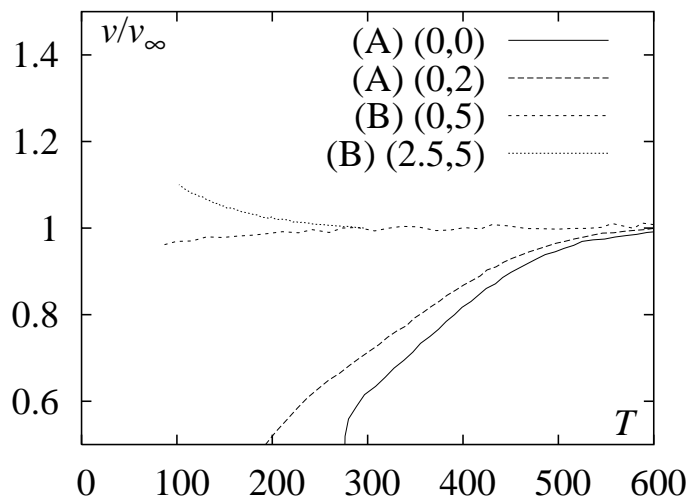

FIG. 9: Dispersion curves of periodic waves, as normalised velocity $v(L) / v(\infty)$ vs time period $T=L / v(L)$, at different parameter values as indicated by the legend. While reaction-diffusion waves go slower if frequent, taxis tends to speed frequent waves up.

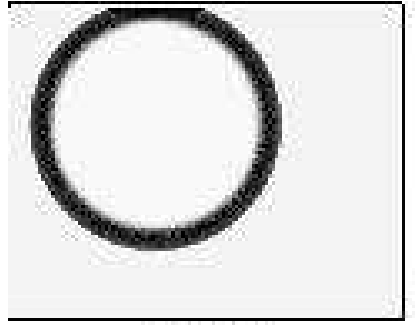

$t=120$

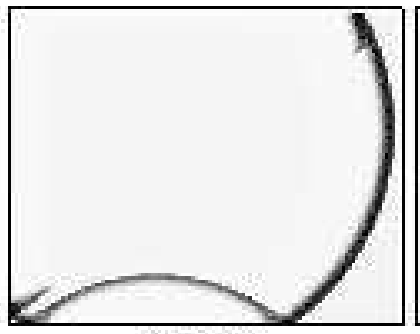

$i=24 k$

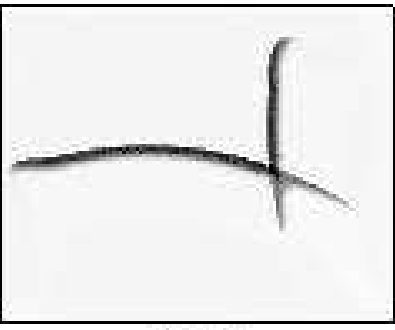

$i=36 \mathrm{k}$

FIG. 10: Circular wave: some parts are reflected from the boundaries, some are not. (B) $200 \times 160(5,1)$. 


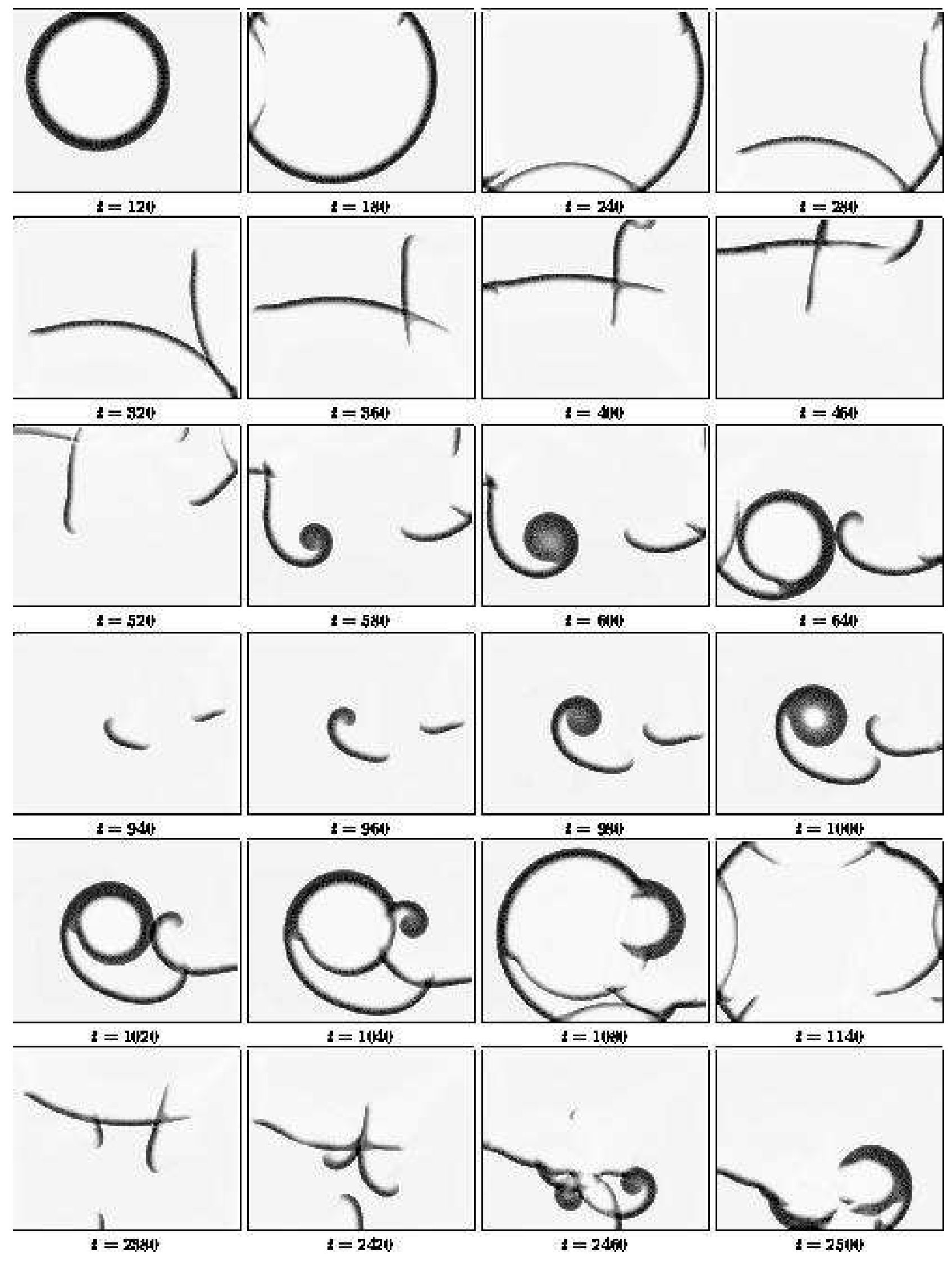

FIG. 11: Self-supporting activity. Continuation of fig. 10 (B) $200 \times 160(5,1)$. 


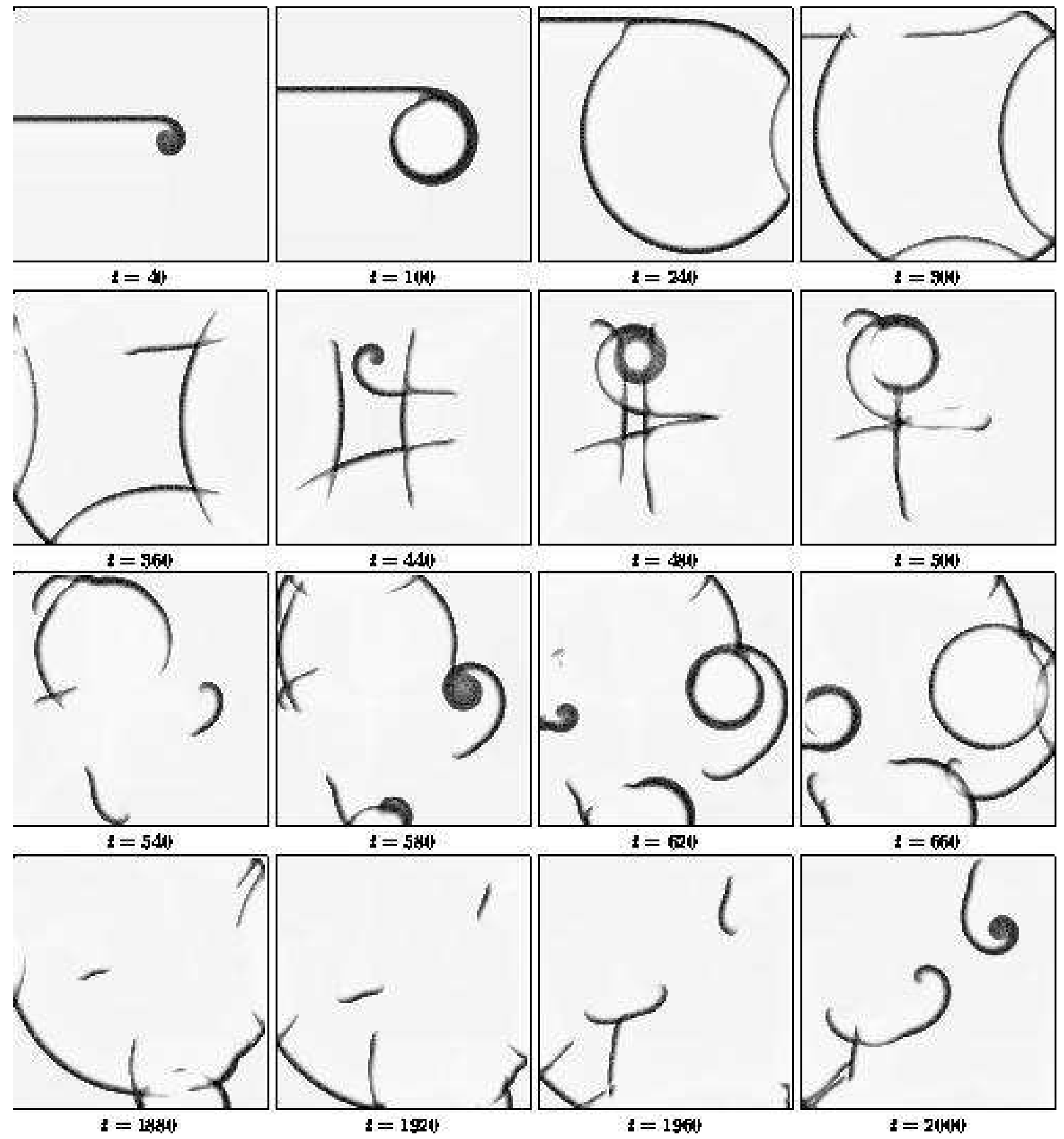

FIG. 12: Self-supporting activity. Continuation of fig. 7 (B) $250 \times 250(5,1)$. 


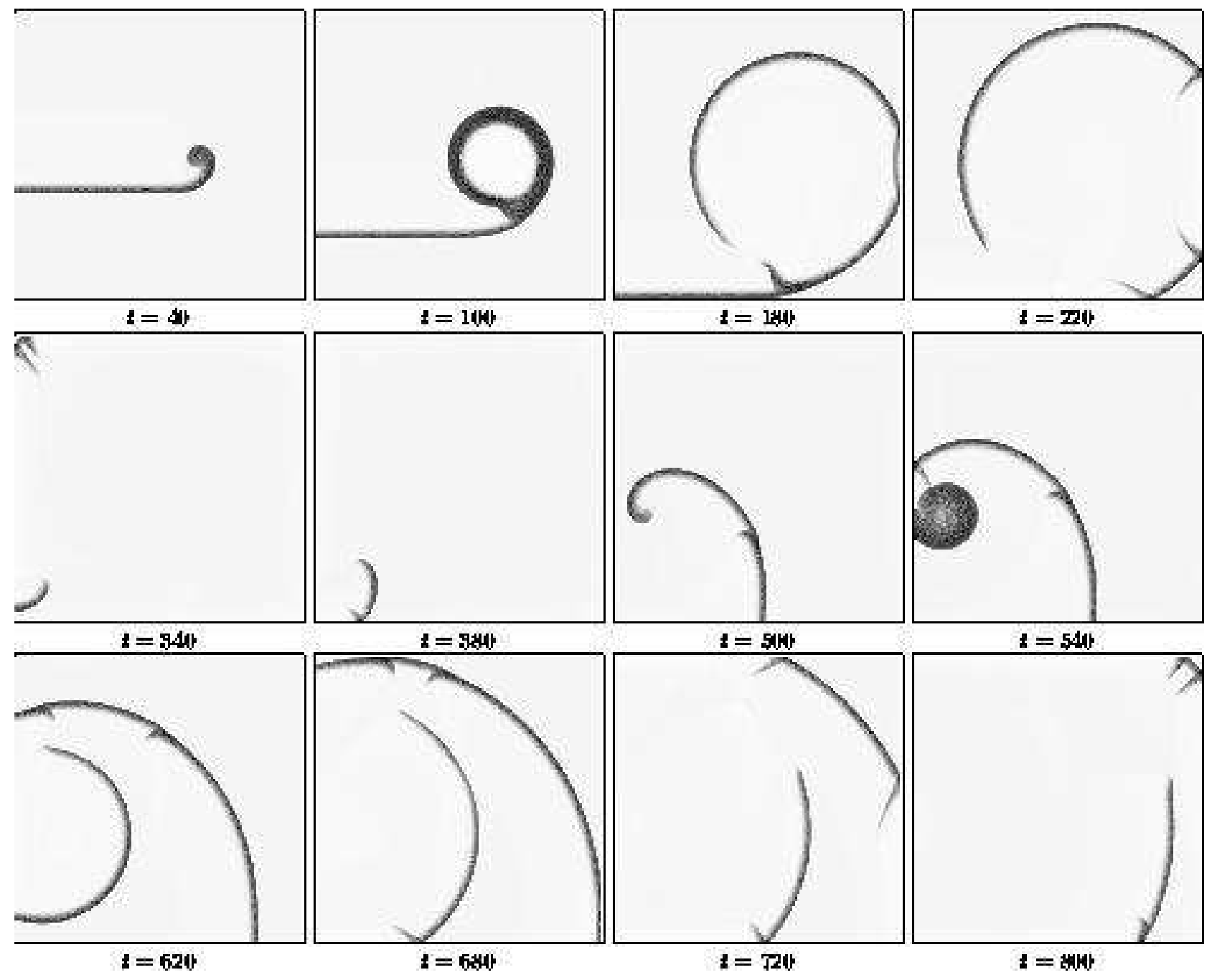

FIG. 13: Self-supporting activity. (B) $250 \times 250(5,2)$. 

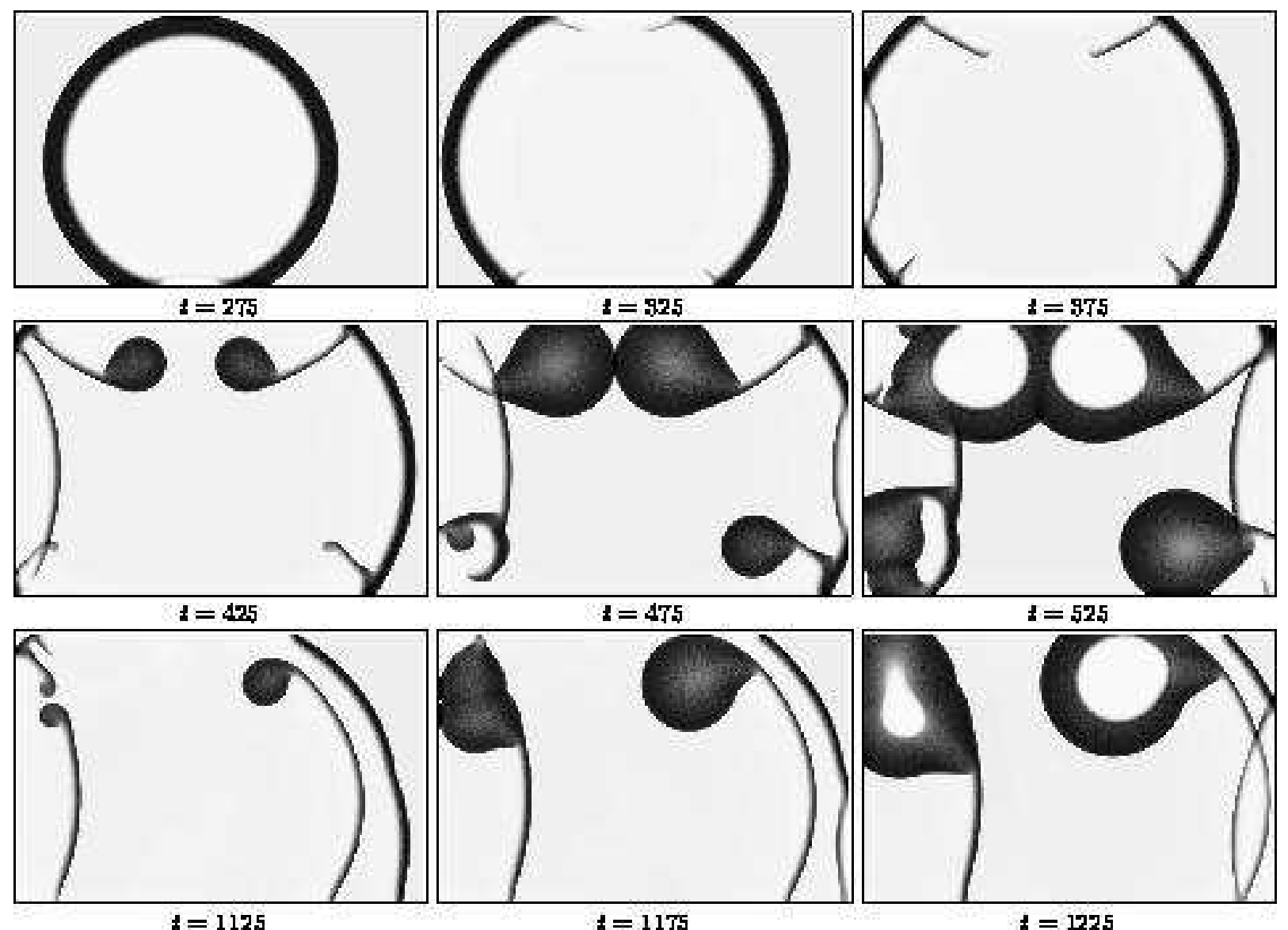

FIG. 14: Self supporting activity, (A) $350 \times 230(2,1.5)$. 


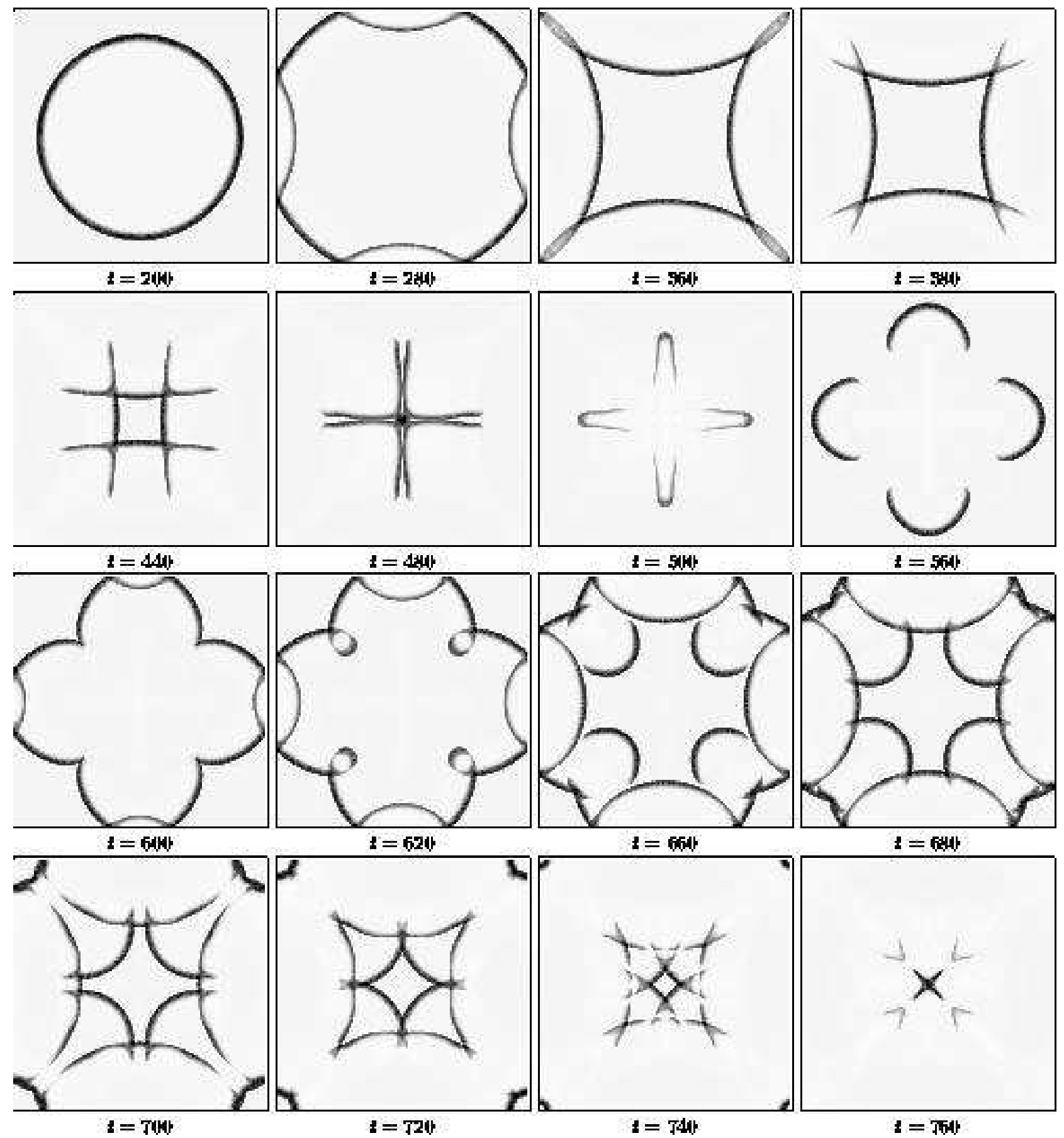

FIG. 15: Self supporting activity, in a symmetric setting. This time the activity stops eventually, when all available waves coincidentally annihilate at the same time. (B) $250 \times 250,(5,1)$. 